\title{
Experiencia de trabajo en equipo: diseño y control de un sistema multivariable de depósitos interconectados
}

\section{Teamwork experience: design and control of a multivariable system of interconnected tanks}

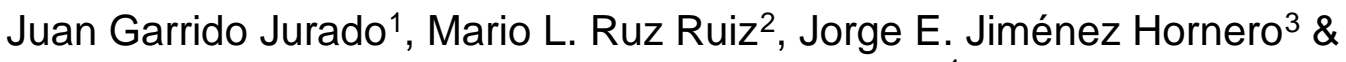 \\ Francisco J. Vázquez Serrano ${ }^{4}$
}

Fecha de recepción: 09/10/2018; Fecha de revisión: 21/01/2019; Fecha de aceptación: 05/04/2019

Cómo citar este artículo:

Garrido, J., Ruz, M.L., Jiménez, J. \& Vázquez, F. (2019). Experiencia de trabajo en equipo: diseño y control de un sistema multivariable de depósitos interconectados. Revista de Innovación y Buenas Prácticas Docentes, 8(2), 56-67.

Autor de Correspondencia: juan.garrido@uco.es

\begin{abstract}
Resumen:
En este trabajo se presenta una experiencia de trabajo en grupo que pretende mejorar tanto competencias generales (organización, planificación, resolución de problemas, toma de decisiones, etc.) como específicas (modelado, diseño e implementación de sistemas de control) del alumnado implicado. Para ello, se propone el montaje e instrumentación por parte de los estudiantes de una planta experimental de tanques interconectados donde se debe controlar el nivel en los tanques actuando sobre un variador de velocidad que impulsa un caudal, y sobre una servoválvula de tres vías que reparte el caudal entre ellos. Después se debe obtener un modelo del sistema para simular y analizar su comportamiento. El proceso diseñado es un sistema de dos entradas y dos salidas que muestra interacción y ello dificulta su control. Se formaron dos grupos de trabajo en los que cada uno tiene que diseñar una estrategia de control multivariable cumpliendo unas especificaciones en el dominio de la frecuencia. Tras simular los diseños propuestos por cada grupo, se validaron con la planta experimental. Finalmente, cada grupo explicó la metodología de trabajo desarrollada. La experiencia de trabajo resultó satisfactoria, destacándose el interés por parte del alumnado en la aplicación de los conceptos teóricos a una planta real.
\end{abstract}

Palabras clave: trabajo en equipo, tecnología de control, simulación, diseño de sistemas.

\footnotetext{
1 Universidad de Córdoba (España), juan.garrido@uco.es; CÓDIGO ORCID: 0000-0001-5122-480

2 Universidad de Córdoba (España), mario.ruz@uco.es; CÓDIGO ORCID: 0000-0002-2356-5253

3 Universidad de Córdoba (España), jjimenez@uco.es; CÓDIGO ORCID: 0000-0001-5560-239X

${ }^{4}$ Universidad de Córdoba (España), fvazquez@uco.es; CÓDIGO ORCID: 0000-0003-4544-6084
} 


\begin{abstract}
:
This work presents a group work experience that aims to improve both general skills (organization, planning, problem solving, decision making, etc.) and specific skills (modelling, design and implementation of control systems) of the students involved. For this, the students had to set the structure and instruments of a proposed experimental plant of interconnected tanks in which the level in the tanks was controlled by acting on a frequency converter that drives a flow, and on a three-way servo valve, which distributes the flow between the two tanks. Then, a system model was obtained to simulate and analyze the system behavior. The designed process is a system of two inputs and two outputs that shows interaction and therefore, its control is more difficult. After that, two working groups were created and each one had to design a multivariable control strategy fulfilling specifications in the frequency domain. After testing the designs proposed by each group in simulation, these were verified with the experimental plant. Finally, each group explained the work methodology developed. The work experience was satisfactory, showing an increase in the interest of students in the application of theoretical concepts to a real system.
\end{abstract}

Key Words: teamwork, control technology, simulation, systems design. 


\section{INTRODUCCIÓN}

El concepto de trabajo en equipo aparece en la mayoría de las guías docentes relacionadas con la Automática ya que un buen ingeniero de control debe aprender a manejar multitud de conceptos, ideas y técnicas y, además, saber aplicarlos a procesos reales de la industria. De ahí que el principal objetivo de la docencia en Automática sea cubrir no solo necesidades de carácter teórico, sino también dotar al alumnado de la capacidad de enfrentarse a problemas de ingeniería. Por otra parte, el trabajo en equipo pretende integrar las habilidades de cada miembro y generar múltiples caminos de aprendizaje, donde el compromiso y la confianza entre los miembros del equipo juegan un papel fundamental (Raposo \& Martínez, 2011). Las experiencias de trabajo en equipo como actividades académicas dirigidas tienen como fin la mejora de varias competencias transversales; de esta manera se intenta mejorar la capacidad de planificación y organización del alumnado y su formación y desarrollo en la resolución de problemas complejos que requieren de grupos de trabajo (Allahverdi \& Aldowaisan, 2015), donde también hay que tener en cuenta habilidades como la comunicación, la resolución de conflictos y el liderazgo (Saad, Robani, Jano \& Makif, 2013; Saad \& Mayid, 2014; Herrera, Muñoz \& Salazar, 2017). El presente trabajo en grupo intenta mejorar las siguientes competencias de los ingenieros:

- Genéricas

o Trabajo en equipo

- Resolución de problemas

o Proporcionar capacidades de abstracción, análisis y síntesis.

- Capacidad de organización y planificación

o Contribuir a la formación y desarrollo del razonamiento científico

- Cognitivas (Saber):

o Diseño e implementación de sistemas de control

o Modelado y análisis de sistemas

- Procedimentales/Instrumentales (Saber hacer):

o Resolución de problemas

- Actitudinales (Ser):

o Habilidad para trabajar de forma autónoma y en equipo

o Toma de decisiones

Por otro lado, la cada vez mayor complejidad de los procesos industriales actuales, en los que el número de magnitudes a controlar es cada vez mayor, obliga al desarrollo de estrategias de control más complejas (Garrido, 2012). Uno de los fenómenos que aparece en los sistemas multivariables es la interacción, por el cual el control sobre una de las variables tiene influencia en el resto de las magnitudes, lo cual es indeseable en la mayoría de los casos (por ejemplo, en una planta mezcladora en la que el aumento de nivel de líquido en un recipiente no debe afectar a la concentración de alguno de los componentes presentes en la mezcla). Para solucionar esta problemática hay diversas metodologías de control multivariable como pueden ser el control descentralizado, control por desacoplo, control robusto, entre otras (Skogestad \& Postlehwaite, 2005).

En la experiencia de trabajo desarrollada se diseña una planta experimental de dos tanques en los que controlar el nivel de agua. Dichos depósitos están interconectados en serie de tal forma que aparezca una interacción entre los actuadores de la planta y dichos niveles, lo cual da al proceso un carácter multivariable. Dicho proceso experimental ha sido montado por el alumnado, el cual, además, ha elaborado diferentes estrategias de control que tratan con el problema de la interacción en sistemas multivariables. La experiencia se ha llevado a cabo principalmente en la asignatura de cuarto curso Laboratorio de Control de Procesos del Grado de Ingeniería Electrónica Industrial. 


\section{DESARROLLO DE LA EXPERIENCIA DE INNOVACIÓN}

Los principales objetivos de la experiencia llevada a cabo, y en los cuales se intenta promover el trabajo en equipo, son los siguientes:

- Diseño y montaje de una planta experimental multivariable de dos depósitos interconectados por parte del alumnado implicado. Dicha planta además ha pasado a formar parte de las plantas experimentales del Laboratorio de Control de Procesos del Área de Ingeniería de Sistemas y Automática de la Universidad de Córdoba para fines docentes.

- Selección y montaje de la instrumentación necesaria para medir los niveles de agua en cada tanque y para poder actuar sobre ellos.

- Elaboración de estrategias de control sobre la planta anterior por diferentes equipos. Ello implicaba el modelado de la planta y el diseño de un controlador.

Para la consecución de los objetivos anteriores se realizaron las siguientes fases:

1. Se estableció un profesor responsable y coordinador de la experiencia.

2. Entre el alumnado, se eligió un coordinador de grupo, que fue el interlocutor con el profesor responsable.

3. En una reunión colectiva se establecieron los requisitos finales del equipo a diseñar.

4. Se seleccionaron los componentes adecuados para montar la planta experimental.

5. Montaje en su conjunto de todos los componentes que conforman el sistema.

6. Finalizado el montaje, se formaron dos equipos, los cuales debían implementar una estrategia de control.

7. Presentación de resultados experimentales de cada uno de los equipos. Cada equipo explicó al resto de compañeros la metodología usada para desarrollar su controlador. De esta forma, los estudiantes comparten entre ellos diferentes experiencias de diseño de controladores y de trabajo en equipo.

\subsection{Diseño y montaje del sistema}

El punto de partida de este trabajo supone el diseño y montaje de un sistema de dos tanques interconectados cuyo esquema se muestra en Figura 1. El sistema está formado por tres tanques rectangulares, una bomba y una servoválvula de tres vías. La bomba toma agua del depósito sumidero y la impulsa a los tanques superiores (tanque 1 y tanque 2). El caudal total $q$ que pasa por la bomba se divide en función de la apertura de la servoválvula en el caudal ql que va al tanque 1 (inferior) y el caudal qh que va al tanque 2 (superior). Además, la bomba se controla mediante un variador de frecuencia lo que permite modificar el caudal total $q$. Así pues, hay dos señales de control: frecuencia del variador (0-100 \%) y porcentaje (0-100\%) de reparto del caudal total a través de la válvula de tres vías que va a la rama superior. Ambas señales afectan a las dos salidas a controlar: los niveles de agua en los tanques 1 y 2 (ambos en $\mathrm{cm}$ ). Esto hace que el proceso sea multivariable, con dos entradas y dos salidas, e interacción entre dichas variables. Los tanques 1 y 2 tienen un sensor de nivel para poder medir las alturas de líquido en ellos ( $h 1$ y $h 2$ ). El líquido en el tanque 1 desagua al depósito sumidero a través de unas válvulas manuales, mientras que el tanque 2 lo hace al tanque 1, lo cual aumenta aún más la interacción del proceso. 
El sistema de control, basado en $\mathrm{PC}$, recibe las medidas de los sensores y regula el variador y la servoválvula mediante diferentes módulos de entrada y salida conectados a una tarjeta de adquisición de datos de National Instruments. Dados unos niveles de referencia en cada tanque, el objetivo es alcanzar dichas referencias con el menor error posible y el menor esfuerzo de control. Dada la naturaleza multivariable de la planta, un cambio en la referencia de nivel de cualquiera de los tanques perturba de forma transitoria al otro debido a la interacción existente. En el PC, la tarjeta de adquisición se comunica con el software Matlab/Simulink® el cual se emplea para diseñar e implementar las estrategias de control. En la Figura 1 se muestra una fotografía de la planta montada por los alumnos.

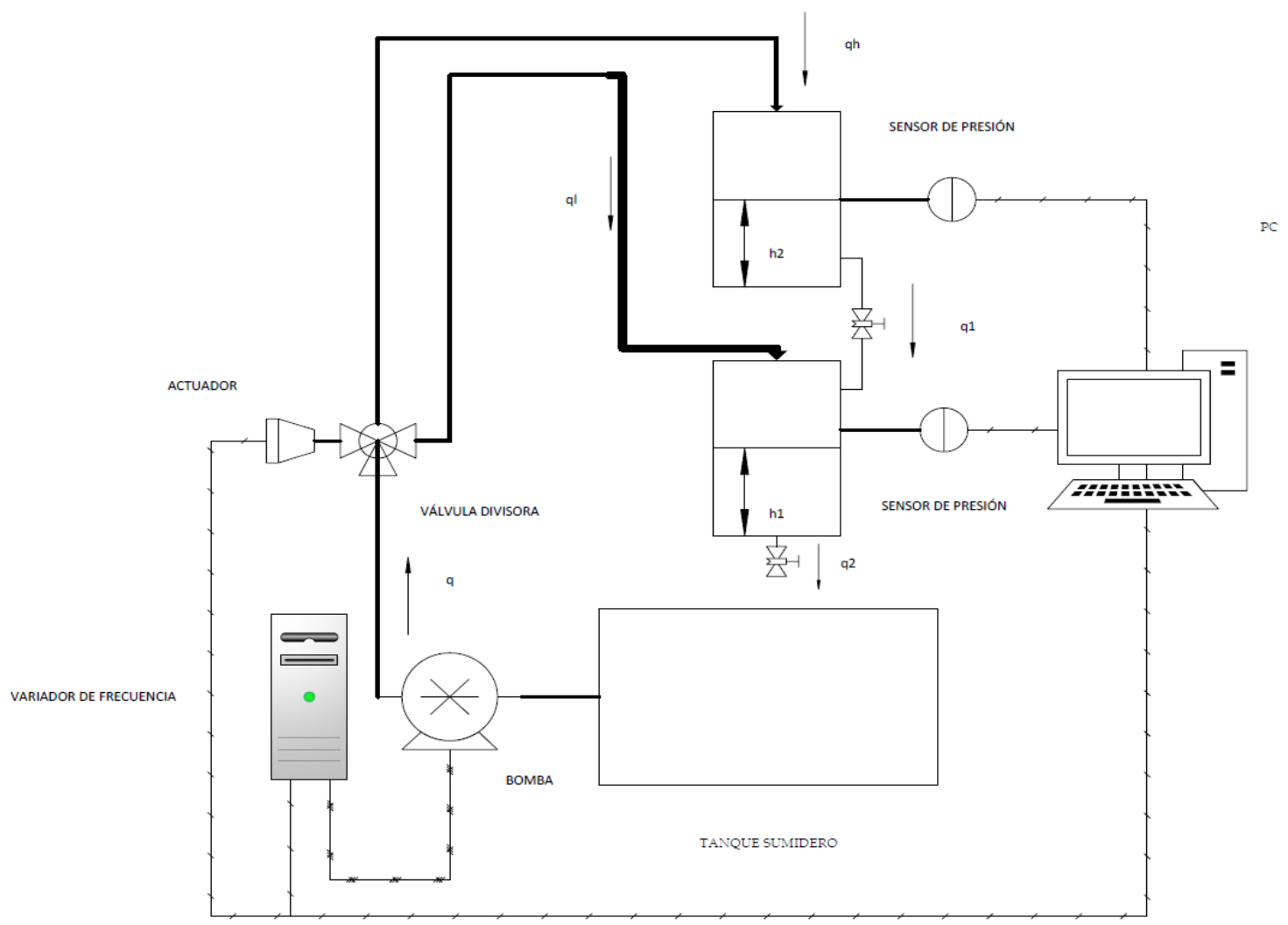

Figura 1. Esquema de la planta. Fuente: Elaboración propia.

\subsection{Modelado e identificación del sistema}

Una vez se han calibrado los instrumentos de medida y actuación, y se ha preparado el sistema de adquisición de datos, antes de proceder a la fase de desarrollo de diferentes estrategias de control es necesario disponer de algún modelo del sistema tanto para el diseño de las estrategias de control como para la simulación de la respuesta obtenida con estas (ver figura 2). 


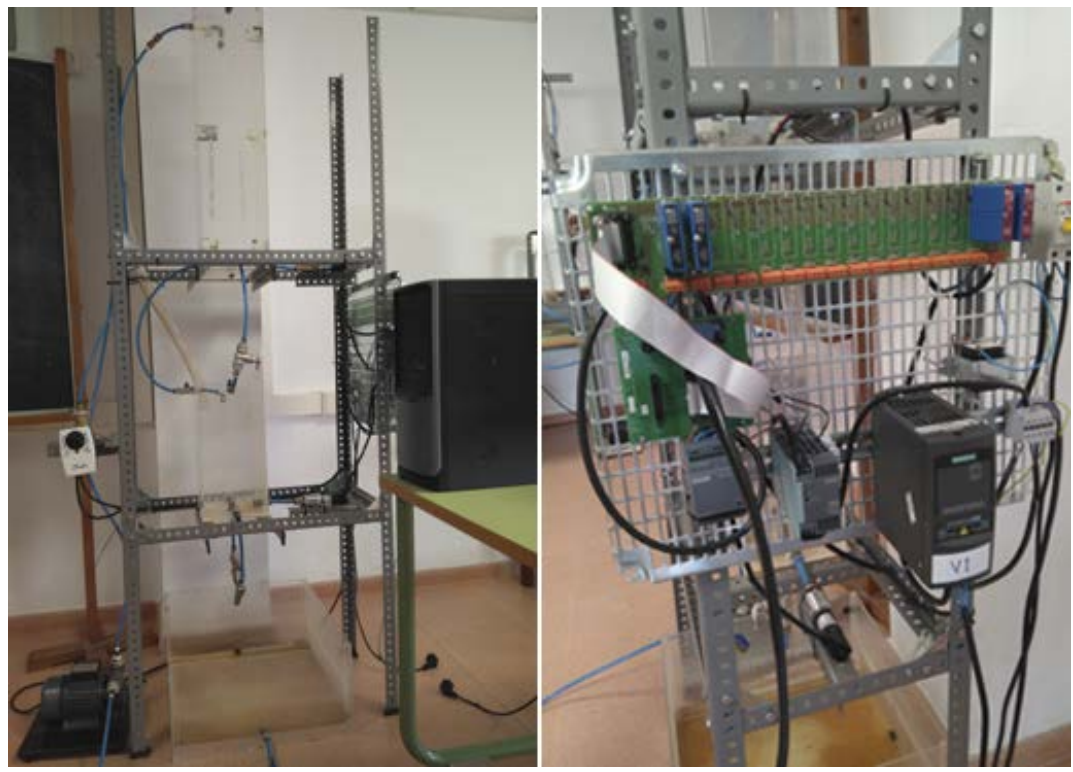

Figura 2. Planta experimental (estructura e instrumentación). Fuente: Elaboración propia.

Para ello, el alumnado realizó una identificación en lazo abierto mediante saltos escalón en las entradas del sistema (frecuencia del variador y apertura de la válvula) y midiendo los niveles en los tanques. A continuación, con la herramienta ident de Matlab obtuvieron funciones de transferencia para cada pareja entrada-salida que se ajustaron a los datos experimentales. El punto de operación en torno al cual se identificó el sistema viene dado por un $65 \%$ en ambas señales de control. El modelo resultante viene dado por la siguiente matriz de funciones de transferencia:

$$
\left(\begin{array}{l}
h_{1} \\
h_{2}
\end{array}\right)=\left(\begin{array}{cc}
\frac{745 s+1.46}{372926 s^{2}+1335 s+1} & \frac{-654 s-0.11}{234639 s^{2}+1160 s+1} \\
\frac{2.4}{853 s+1} & \frac{2}{864 s+1}
\end{array}\right) \cdot\left(\begin{array}{l}
u_{\text {bomba }} \\
u_{\text {valvula }}
\end{array}\right)
$$

En la figura 3 se muestra la respuesta experimental del sistema en lazo abierto ante un salto escalón del $10 \%$ en la señal de control de la bomba, así como la respuesta en simulación con el modelo identificado. En la figura 4 se muestra la respuesta, también en lazo abierto, del sistema y del modelo cuando el salto escalón del $10 \%$ se realiza en la válvula. El ajuste del nivel del tanque 1 ( $h 1$ ) es muy bueno en ambos casos; el de $h 2$ es algo peor y la medida de este nivel contiene bastante ruido debido al rango del sensor de nivel usado. Aun así, el modelo obtenido tiene la misma tendencia que el sistema real y es válido para el diseño de controladores. En ambas experiencias se puede apreciar que las dos entradas tienen efecto en los dos niveles de agua a controlar, lo cual reafirma la presencia de interacción en este sistema multivariable. 


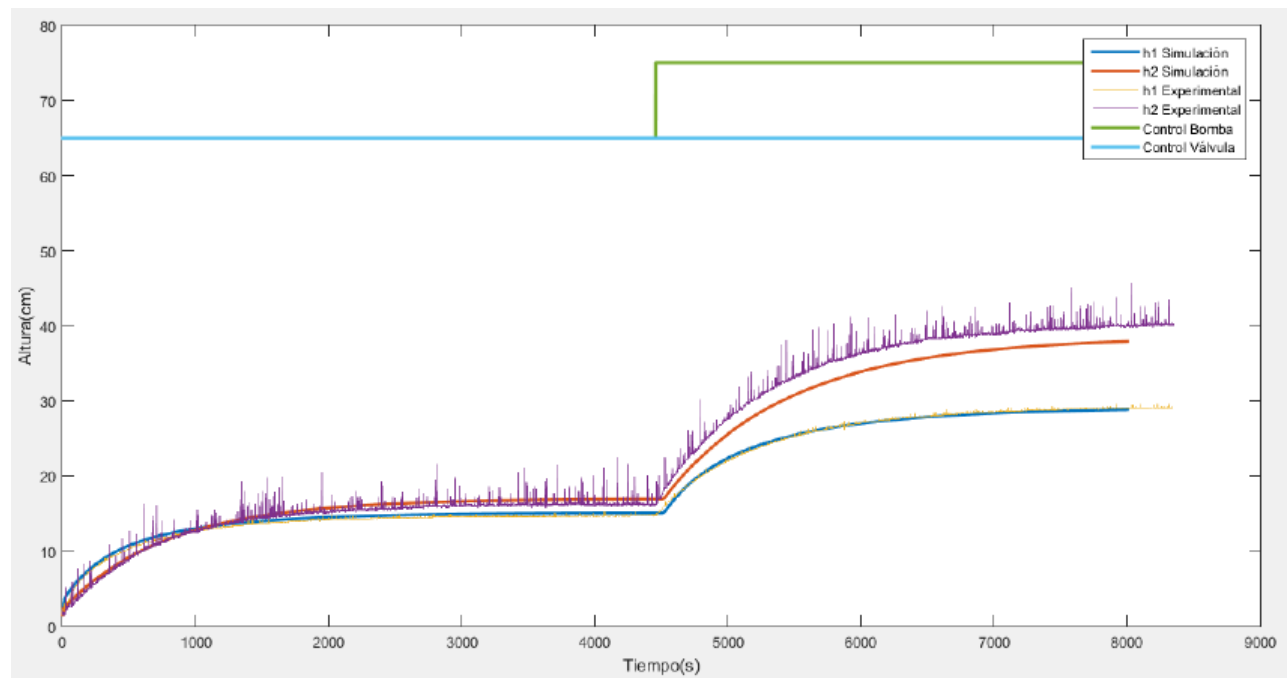

Figura 3. Alturas h1 y h2 en lazo abierto (experimentales y simulación) ante salto en la bomba. Fuente: Elaboración propia.

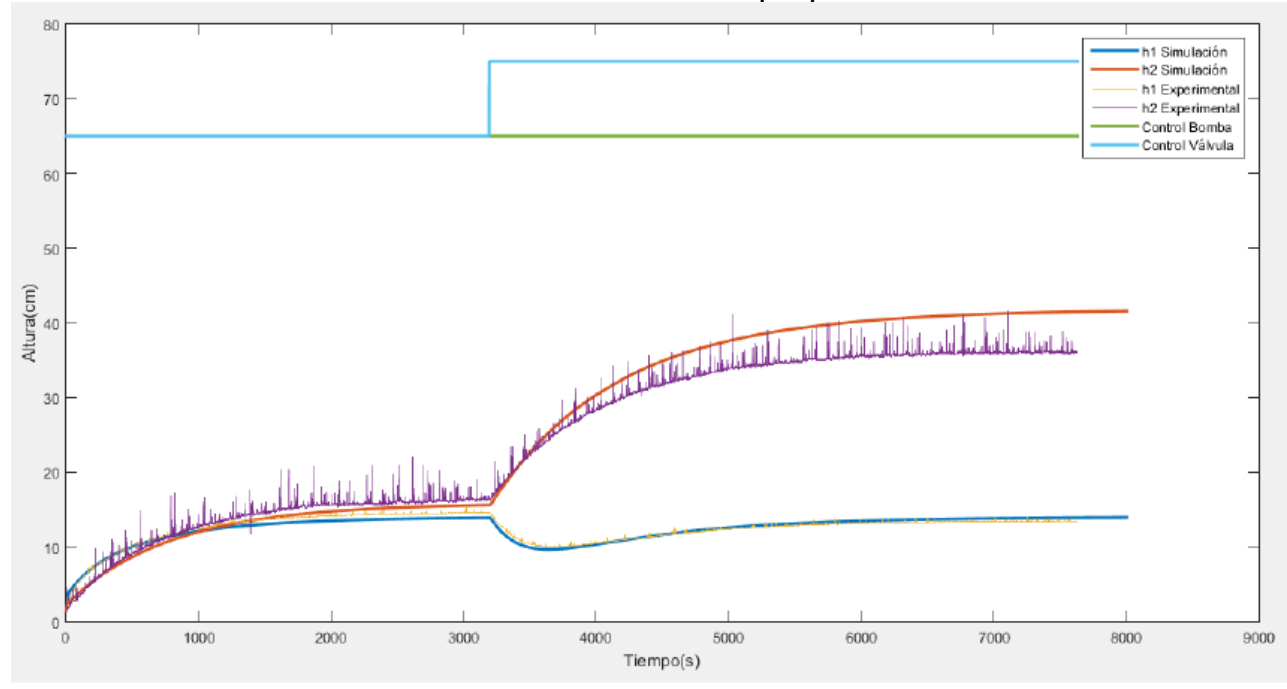

Figura 4. Alturas h1 y h2 en lazo abierto (experimentales y simulación) ante salto en la servoválvula. Fuente: Elaboración propia.

\subsection{Diseño de las estrategias de control en lazo cerrado}

Una vez obtenido un modelo del sistema a controlar, se formaron dos equipos de alumnos para que cada uno diseñara una estrategia de control multivariable diferente, en concreto un control descentralizado y un control por desacoplo simplificado. A continuación, se detallan cada una de ellas.

\subsubsection{Control descentralizado}

En una estrategia descentralizada, cada variable controlada se regula mediante una variable manipulada de manera independiente, sin que el controlador responsable reciba información del resto del proceso. La figura 5 muestra un esquema de control descentralizado. Por tanto, el primer paso es determinar con qué variables manipuladas se va a controlar cada salida, esto es, el problema de emparejamiento entradas-salidas. Para ello una herramienta útil es la matriz de ganancias relativas (RGA), la cual aconseja emparejamientos cuyos elementos correspondientes en dicha matriz estén próximos a la unidad (Bristol, 1966). La RGA del modelo del sistema viene dada en (2), la cual recomienda emparejar la altura del tanque 1 con la frecuencia del variador, y el nivel del tanque 2 , con la servoválvula. 


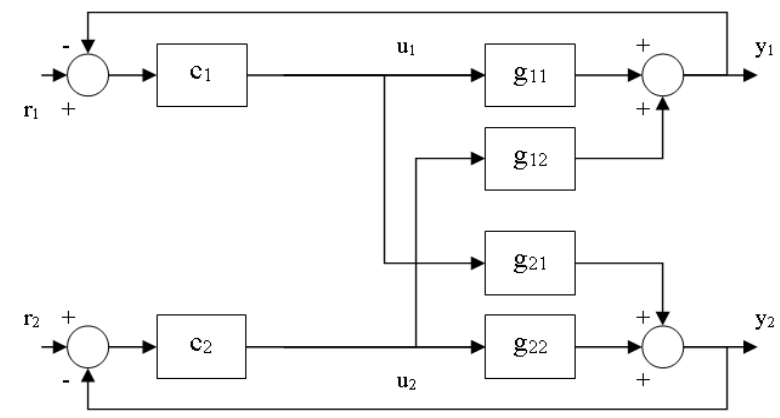

Figura 5. Esquema de control descentralizado. Fuente: Elaboración propia.

$$
R G A=\left(\begin{array}{ll}
0.92 & 0.08 \\
0.08 & 0.92
\end{array}\right)
$$

Tras realizar todo este análisis, el grupo responsable decidió usar controladores PI (proporcional-integral) para cerrar los lazos. Un control PI proporciona una señal de control que es proporcional al error actual de la magnitud a controlar y proporcional a la integral del error (Aström \& Hägglund, 2006). Su expresión viene dada en (3), en la cual hay dos parámetros a ajustar: la ganancia proporcional Kp y la constante de tiempo integral Ti.

$$
u(t)=K p \cdot e(t)+\frac{K p}{T i} \int e(t) \cdot d t
$$

Para ajustar los parámetros de los dos controladores PI, el alumnado utilizó una metodología iterativa implementada en la herramienta TITO, en la que usó un margen de $60^{\circ}$ como especificación en ambos lazos. La herramienta TITO, elaborada por profesores del área de Ingeniería de Sistemas y Automática (Vázquez, Morilla \& Dormido,1999), ajusta los controladores teniendo en cuenta las influencias entre ambos lazos de control para mejorar la respuesta con respecto a una sintonía individual. En la Tabla 1 se muestran los parámetros obtenidos para el lazo 1 (h1-frecuencia bomba) y lazo 2 (h2-válvula). Antes de probar el diseño con la planta real se realizó una simulación con el modelo de la planta para comprobar su respuesta. La Figura 6 muestra dichos resultados en simulación. Se ha llevado a cabo un salto de $5 \mathrm{~cm}$ en la referencia de cada nivel en instantes de tiempo diferentes. Puede apreciarse que, ante estos cambios, las nuevas referencias se alcanzan, pero apareciendo algo de interacción en el lazo de control contrario, la cual es rechazada rápidamente por su controlador correspondiente.

Tabla 1.

Parámetros de los controladores.

\begin{tabular}{lcc} 
& Control descentralizado & Control con desacoplo \\
\hline Kp1 & 4.6 & 2.2 \\
\hline Kp2 & 4 & 1.9 \\
\hline Ti1 & 314 & 68 \\
\hline Ti2 & 368 & 72 \\
\hline
\end{tabular}

Fuente: Elaboración propia. 


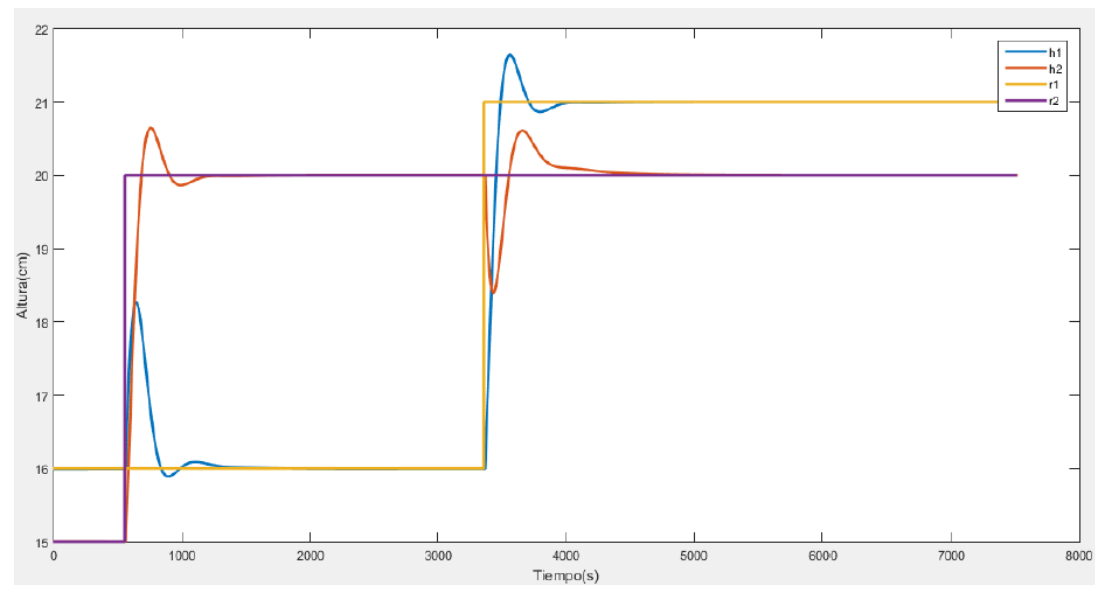

Figura 6. Simulación del control descentralizado. Fuente: Elaboración propia.

\subsubsection{Control por desacoplo simplificado}

Esta metodología combina una red de desacoplo con un control descentralizado (Figura 7). La red de desacoplo $D(s)$ intenta eliminar o reducir la interacción del proceso, de tal manera que el conjunto red desacoplo más planta se comporte como dos sistemas independientes. De esta forma, cada uno de los controladores del control descentralizado se puede sintonizar de manera totalmente independiente (Wang, 2003).

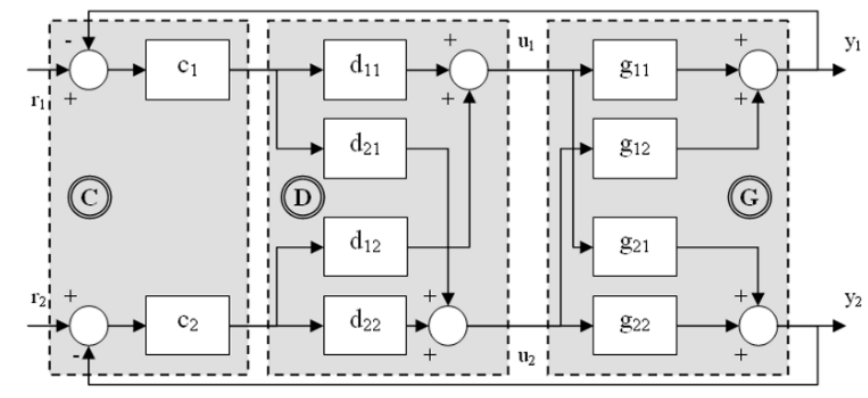

Figura 7. Esquema de control descentralizado más red de desacoplo. Fuente: Elaboración propia.

Hay diferentes tipos de redes de desacoplo. En este caso los alumnos decidieron utilizar el desacoplo simplificado por la simplicidad de sus elementos, ya que los elementos de su diagonal son igual a la unidad (Garrido, Vázquez \& Morilla, 2012). La red resultante se muestra en la ecuación (4).

$$
D=\left(\begin{array}{cc}
1 & \frac{-(654 s+0.11)(864 s+1)}{2\left(234639 s^{2}+1160 s+1\right)} \\
\frac{2.4\left(372926 s^{2}+1335 s+1\right)}{(853 s+1)(745 s+1.46)} & 1
\end{array}\right)
$$

Tras ello, ajustaron dos controladores PI para los dos procesos desacoplados (planta más desacoplo) usando como especificación un margen de fase de $60^{\circ}$ en ambos lazos, de manera similar al caso de la estrategia puramente descentralizada. Los parámetros resultantes aparecen también recogidos en la Tabla 1, y la respuesta en lazo cerrado se muestra en la Figura 8. Ahora la respuesta ante los cambios en las referencias es similar al caso descentralizado, pero, debido a la red de desacoplo, ya no aparece interacción en el lazo contario. 


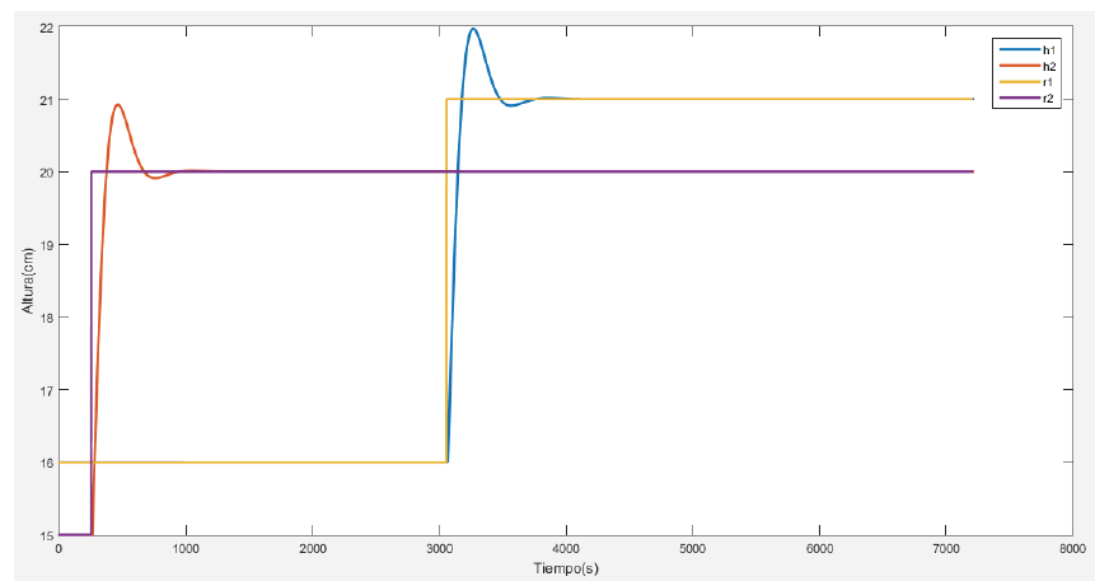

Figura 8. Simulación del control descentralizado con red de desacoplo. Fuente: Elaboración propia.

\section{RESULTADOS}

A continuación, los diseños desarrollados por cada equipo se implementaron y verificaron en el sistema real llevando a cabo una prueba similar a la de simulación, esto es, mismo punto de operación y mismos saltos en las referencias. Los resultados experimentales se muestran en las figuras 9 y 10. En ambos casos, el sistema se comporta cualitativamente de forma similar a la simulación. Bajo el control descentralizado, la respuesta es muy similar. En el caso del control por desacoplo simplificado, sigue apareciendo cierta interacción entre los lazos debido a los errores de modelado y las incertidumbres del sistema. Sin embargo, dicha interacción es menor en comparación con el caso descentralizado. En ambos experimentos, también se aprecia una mayor oscilación del nivel $h 2$ con respecto a la simulación. Esto es debido a efectos no lineales de la servoválvula de tres vías que no se tuvieron en cuenta en la fase de modelado.

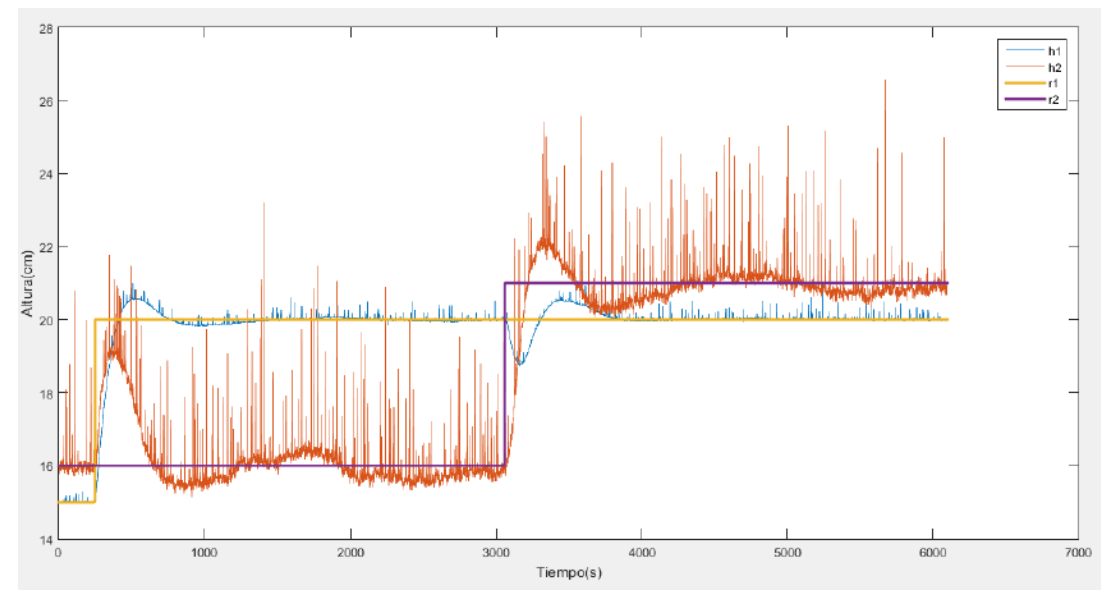

Figura 9. Resultados experimentales del control descentralizado. Fuente: Elaboración propia. 


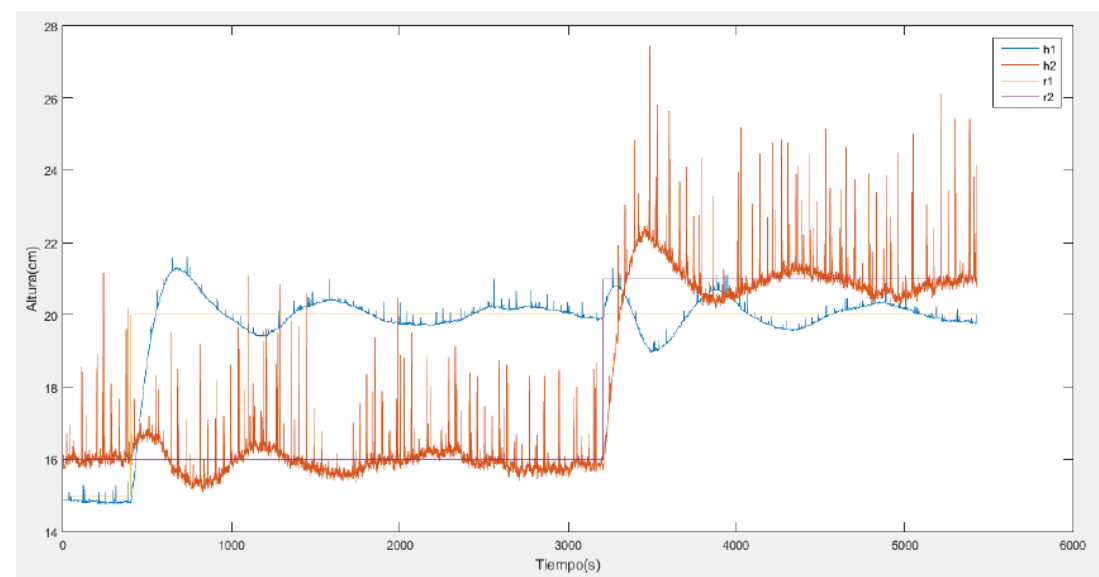

Figura 10. Resultados experimentales del control descentralizado con red de desacoplo. Fuente: Elaboración propia.

Ambos equipos implementaron las correspondientes estrategias de control de forma adecuada, siguiendo la metodología y fases de desarrollo explicadas por el profesorado. No se apreciaron diferencias significativas entre grupos en lo que a consecución de objetivos se refiere, y los alumnos de ambos grupos trabajaron de forma adecuada. Una vez finalizadas y validadas en la planta experimental las estrategias de control descentralizado y por desacoplo simplificado, el profesor responsable reunió a ambos grupos, resaltando las ventajas que pueden suponer el uso de estrategias de control multivariable. Los alumnos de ambos grupos mostraron especial interés al comprobar experimentalmente la disminución de la interactividad mediante el uso de la estrategia de control por desacoplo simplificado.

\section{CONCLUSIONES}

La experiencia de trabajo en equipo presentada en este trabajo y que ha implicado a varios estudiantes del Grado de Ingeniería en Electrónica Industrial ha potenciado la mejora en competencias:

- Mejora de competencias generales como la toma de decisiones, organización y planificación mediante el diseño y construcción de la planta real trabajando en grupo.

- Mejora en competencias específicas de la Automática (conocimiento del modelado, técnicas de control y aplicación a la automatización industrial) mediante la motivación de los estudiantes y una mejor compresión de las principales etapas en el análisis y diseño de un problema de control: instrumentalización de la planta, modelado del sistema, diseño de la estrategia de control, simulación hasta alcanzar las especificaciones requeridas, implementación del controlador, experimentación real, etc.

Además, de manera más concreta, el trabajo profundiza en aspectos más avanzados de control como los sistemas multivariables y su problemática de interacción entre variables, y el diseño de diferentes estrategias de control multivariables. En cuanto al desarrollo de la experiencia de trabajo, se pudo observar un especial interés del alumnado en la comprobación de los resultados de simulación de forma experimental (fase de validación). Finalmente, se ha obtenido una planta experimental operativa que pueda servir para prácticas en futuros cursos de las asignaturas implicadas. 


\section{REFERENCIAS}

Allahverdi, A. \& Aldowaisan. (2015). T. Assessment of student outcomes of the Industrial and Management Systems Engineering programme at Kuwait Universit. Global Journal of Engineering Education, 17(3), 103-112.

Aström, K. Hägglund, T. (2006). Advanced PID Control. North-Carolina: ISA.

Bristol, E. H. (1966). On a New Measure of Interaction for Multivariable Processes Control. IEEE Transactions on Automatic Control, 11(1), 133-134. DOI: https://doi.org/10.1109/TAC.1966.1098266

Garrido, J., Vázquez, F. y Morilla, F. (2012). Centralized multivariable control by simplified decoupling. Journal of Process Control, 22(6), 1044-1066. DOI: https://doi.org/10.1016/j.jprocont.2012.04.008

Garrido, J. (2012). Diseño de sistemas de control multivariable por desacoplo con controladores PID. Tesis Doctoral. Madrid: UNED.

Herrera, R.F., Muñoz-La Rivera, F.C. \& Salazar. L.A. (2017). The development's perception of teamwork competency in the formation of undergraduate engineering students. Global Journal of Engineering Education, 19(1), 30-35.

Raposo, M. \& Martínez E. (2011). La Rúbrica en la Enseñanza Universitaria: Un Recurso para la Tutoría de Grupos de Estudiantes. Formación Universitaria, 4(4), 19-28.

Saad, M.S. y Majid, I.A. (2014). Employers' perceptions of important employability skills required from Malaysian engineering and information and communication technology (ICT) graduates. Global Journal of Engineering Education, 16(3), 110-115.

Saad, M.S., Robani, A., Jano, Z. \& Makif, I.A. (2013). I.A. Employers' perception on engineering, information and communication technology (ICT) students'employability skills. Global Journal of Engineering Education, 15(1), 4247.

Skogestad, S., Postlehwaite, I. (2005). Multivariable Feedback Control: Analysis and Design. England: Wiley.

Vázquez, F., Morilla, F. \& Dormido, S. (1999). An iterative method for tuning decentralized PID controllers, en Proceedings of the 14th IFAC World Congress, Beijing, China.

Wang, Q. G. (2003). Decoupling Control. Berlin-Heidelberg: Springer. 